\title{
RDUS
}

Revue de DROIT

UNIVERSITÉ DE SHERBROOKE

Titre : $\quad$ PERSPECTIVES POUR UN PRINCIPE DE SÉCURITÉ JURIDIQUE EN DROIT CANADIEN : LES PISTES DU DROIT EUROPÉEN

Auteur(s): $\quad$ Martin NADEAU

Revue : $\quad$ RDUS, 2009-2010, volume 40, numéro 1-2

Pages: $\quad 511-550$

ISSN : $\quad 0317-9656$

Éditeur : Université de Sherbrooke. Faculté de droit.

URI : $\quad$ http://hdl.handle.net/1 $\quad$ 1143/10459

DOI : https://doi.org/10.17118/11143/10459 
Page vide laissée intentionnellement. 


\title{
ARTICLE
}

\section{PERSPECTIVES POUR UN PRINCIPE DE SÉCURITÉ JURIDIQUE EN DROIT CANADIEN : LES PISTES DU DROIT EUROPÉEN}

\author{
par Martin NADEAU*
}

La sécurité juridique dans le discours juridique européen a d'abord été conçue ou présentée comme une valeur ou un objectif du droit et était principalement l'objet d'études en théorie et en philosophie du droit. Progressivement, le discours juridique européen a évolué vers une reconnaissance de son statut formel et est alors devenu aux yeux des auteurs, un principe, une norme juridique. Particulièrement, la Cour européenne des droits de l'homme et la Cour de justice de la Communauté européenne ont reconnu et appliqué dans leur jurisprudence un principe général de la sécurité juridique. La doctrine a, par la suite, fait écho à la jurisprudence et a affirmé qu'il y a eu, sous son impulsion, émergence et consécration d'un principe de sécurité juridique dans certains droits nationaux et en droit supranational européen. Mais certains auteurs vont plus loin en soutenant que la sécurité juridique est une norme universelle inhérente à l'État de droit et s'imposerait aux juges et législateurs. La sécurité juridique, concept à contenu variable, se révèle être une norme polymorphe et adaptable qui fédère les exigences propres à chaque système juridique et dont l'autonomie est problématique.

$A u$ Canada, les discours juridictionnel et doctrinal ont récemment introduit le concept de sécurité juridique, mais ne lui reconnaissent pas expressément un statut de norme juridique. L'exemple des juridictions européennes illustre le potentiel juridique du principe, c'est-à-dire son influence sur la pensée juridique et sur l'élaboration des règles de droit. Cette mise en forme discursive dans le discours juridique canadien rend probable, mais hypothétique, une reconnaissance à terme par les juristes d'un statut de principe à ce qui demeure pour l'instant un objectif ou une valeur du droit canadien ${ }^{* *}$.

\footnotetext{
* Avocat, ministère de la Justice du Québec, soutien à la magistrature, Cour supérieure du Québec.

** L'auteur a réalisé un mémoire sur le concept de sécurité juridique (Martin Nadeau, Sur les traces d'un principe de sécurité juridique en droit canadien. Les pistes du droit européen, mémoire de maîtrise en droit, sous la direction de Mathieu Devinat, Université de Sherbrooke, 2009 [non publié]).
} 
In European juridical discourse, legal certainty was initially conceptualized as a legal value or objective and, for the most part, was a subject of research in the field of legal theory and philosophy. European legal discourse gradually recognized the formal status of legal certainty, which subsequently became a principle or legal norm. The European Court for Human Rights and the Court of Justice of the European Communities, in particular, have recognized and now apply a general principle of legal certainty in their case law. Considering this, legal scholarship now states that the principle of legal certainty in domestic and supranational European law has emerged and has been officially enshrined. But some writers go further by arguing that legal certainty is a universal norm inherent in the rule of law and binding on judges and legislators. The multifaceted concept of legal certainty has now become a versatile, malleable standard that dictates the specific requirements of each judicial system whose autonomy is problematic.

In Canada, the concept of legal certainty was recently introduced in jurisdictional and doctrinal discourse, without, however, receiving express status as a legal norm. The European example illustrates the concept's legal potential, in other words, its impact on judicial thought and on the implementation of laws. The Canadian discursive approach to the concept makes it likely, yet hypothetical, that legislators will eventually confer on legal certainty, which is currently a legal value and objective, the status of a principle. 
Perspectives pour un principe de sécurité juridique

(2009-10) 40 R.D.U.S. en droit canadien :

les pistes du droit européen

\section{SOMMAIRE}

Introduction

Section I : Un débat à l'ombre des règles : la sécurité juridique comme objectif du droit

Section II : Émergence du principe de sécurité juridique dans le discours juridique européen ............... 533

Section III : Émergence dans le discours juridique canadien du concept de sécurité juridique ...... 540

A. Le discours doctrinal............................. 541

B. Le discours du juge de la Cour suprême

Conclusion générale 

Perspectives pour un principe de sécurité juridique

(2009-10) 40 R.D.U.S. en droit canadien :

515

les pistes du droit européen

\section{Introduction}

Définir le sens attribué par la doctrine européenne au concept de sécurité juridique est une entreprise périlleuse. Les " contours assez fluctuants " du concept de sécurité juridique font que "chacun peut s'en faire sa propre idée "1. "Il paraît donc malaisé de concevoir la notion en soi, de manière générique ou abstraite " 2 parce qu'il s'agit d'un concept hétéronyme aux multiples statuts (règle de droit positif, valeur éthique ou philosophique, objet d'étude). Malgré l'impossibilité de constituer une définition "parfaite" de la sécurité juridique, il n'est pas inutile de donner une "définition téléologique " de la sécurité juridique "qui repose essentiellement sur une recherche des consensus de la doctrine ${ }^{3}$. Cette définition est purement conventionnelle ${ }^{4}$, mais elle permet de cerner les buts poursuivis par les tenants de la sécurité juridique et le sens qui lui est généralement attribué.

1. Hubert Groutel, "Exposé introductif " (1998) 3 R.G.D.A. dans Insécurité juridique et assurance à la p. 419 .

2. Sylvia Calmes, Du principe de protection de la confiance légitime en droit Allemand, Communautaire et Français, thèse de doctorat en droit public, sous la direction de Didier Truchet, Université Pantheon-Assas (Paris II), 2000 [non publiée] à la p. 148 [Calmes, thèse].

3. Anne-Laure Valembois, La constitutionnalisation de l'exigence de sécurité juridique en droit français, Paris, L.G.D.J., 2005 à la p. 13.

4. Drieu Godefridi, "État de droit, liberté et démocratie " (2004) $23 \mathrm{n}^{\circ} 1$ Politique et Sociétés à la p. 144: "toute théorie du droit repose nécessairement sur des élucidations minimales de termes. Sans doute Karl Popper a-t-il raison d'écrire que " nous devrions absolument éviter, comme la peste, de discuter le sens des mots" et de récuser toute question du type "Qu'est-ce que? " parce que purement linguistique et donc stérile (Karl Popper, La connaissance objective, Paris, Aubier, 1991 aux pp. 10, 456). Les mots sont des conventions. Seule la réalité, leur dénotation, doit nous importer. Mais il n'en reste pas moins que, d'une part, pour conventionnels qu'ils soient, les mots sont notre seul accès à la réalité et que, d'autre part, pour que des mots disputés puissent être qualifiés de “ conventions", il faut en préciser les termes. Un dialogue entre deux interlocuteurs incapables de s'entendre sur ne serait-ce qu'une définition partielle des termes utilisés équivaut à un dialogue téléphonique entre un Chinois unilingue et un Luxembourgeois unilingue, c'est-à-dire à une absence de dialogue ". 
La sécurité juridique dans sa définition téléologique renvoie à l'idée d'une série d'exigences que doivent posséder soit les normes juridiques, soit le système pris en tant que tel ${ }^{5}$. Ainsi, "[1]'exigence [de sécurité juridique] a, à son tour, ... des exigences " 6 . D’abord, la sécurité juridique se décrit comme l'exigence de la prévisibilité du droit, à cet égard, les règles de droit ne doivent régir que les actions futures afin de permettre aux justiciables de prévoir les conséquences juridiques de leurs actes et de leurs actions (ex: principe de non-rétroactivité, "rule of law ", règles de droit transitoire). Ensuite, dans l'optique de savoir afin de prévoir, la sécurité juridique présuppose l'accessibilité aux normes juridiques. Au plan formel, cela requiert la publication des normes juridiques. Au plan matériel, l'accessibilité exige que les justiciables soient en mesure de comprendre les normes juridiques (ex. : principe de précision des lois, intelligibilité des motifs des jugements). Enfin, les justiciables doivent pouvoir compter sur leurs prévisions lorsqu'ils actualisent une action dans la durée, ce qui exige la stabilité du droit (ex. : respect des attentes légitimes, délai de prescription, délai de recours, publicité des droits, autorité de la chose jugée, règle du précédent, nullité prospective d'une loi inconstitutionnelle). La sécurité juridique est donc, selon le statut que les juristes lui accordent et les contextes, un concept, un idéal ou un principe qui exige la prévisibilité, la stabilité et l'accessibilité aux règles ${ }^{7}$.

La sécurité juridique n'est pas une norme prévue expressément dans les textes législatifs ou constitutionnels par les ordres juridiques canadiens et européens. Soutenir que la sécurité juridique est un principe de droit positif revient à prendre position

5. Caroline Salviejo, Le principe de sécurité juridique en droit communautaire et européen, thèse de doctorat en droit public, sous la direction de Frédéric Sudre, Université de Montpellier I, 2003 [non publiée] à la p. 2.

6. Sylvie Ciamonti, "Sécurité juridique et mécanismes d'application du droit dans le temps " (2008) 110 R. du N. 631 à la p. 633.

7. Certains auteurs y ajoutent une dimension spatiale. Pour une étude exhaustive sur cette question: Philippe Raimbault, Recherche sur la sécurité juridique en droit administratif français, t. 256, Paris, L.G.D.J., 2009 aux pp. 197-352. 
Perspectives pour un principe de sécurité juridique

(2009-10) 40 R.D.U.S. en droit canadien :

les pistes du droit européen

sur son statut juridique formel controversé8. En effet, "[1]a norme créée par le juge [et la doctrine] ne bénéficie pas d'une formulation arrêtée " et affirmer que la sécurité juridique est une norme juridique valide parce qu'elle est créée par le juge [ou reconnue par la doctrine], cela sous-tend "un processus de sélection d'information par le juriste qui s'appuie sur une méthode lorsqu'il identifie et interprète une règle dérivée de la jurisprudence [ou de la doctrine] $»^{9}$.

Nous n'utilisons pas la notion de sécurité juridique pour désigner une norme juridique ou un principe dans tous les cas, et ce, afin de ne pas accorder a priori un statut de norme juridique formelle à la sécurité juridique. Notre objectif est de présenter le statut que les auteurs accordent à la sécurité juridique et d'analyser les fonctions de la sécurité juridique dans les discours juridiques ${ }^{10}$. Pour cette raison, la présente étude n'a pas pour objet de s'interroger sur la nature juridique du principe de sécurité juridique. Elle cherche plutôt à dégager le sens qu'il revêt au sein du discours juridique, "c'est-à-dire le sens que cette

8. Voir par ex. Ingrid Fournol, Le principe de sécurité juridique en droit communautaire et en droit administratif français, thèse de doctorat en droit public, sous la direction de Jacqueline Dutheil de la Rochère, Université Pantheon-Assas (Paris II), 1999 [non publiée] à la p. 358 : l'interprétation des jugements de la CJCE ne permet pas de donner " une qualification juridique indiscutable à la sécurité juridique ".

9. Mathieu Devinat, La règle prétorienne en droit civil français et dans la common law canadienne. Étude de méthodologie juridique comparée, Aixen-Provence, Presses Universitaires d'Aix-Marseille, 2005 à la p. 6; Philippe Malaurie, "Rapport français " dans La réaction de la doctrine à la création du droit par les juges, Paris, Economica, 1982 à la p. 83 : "Le jugement parle un langage qui ne se lit pas immédiatement comme une règle de droit; c'est à la doctrine que revient la tâche de formuler la règle, reliant les différents jugements, mettant en relief les limites et les exceptions à la règle énoncée ".

10. Comparer Philippe Raimbault, "La sécurité juridique, nouvelle ressource argumentative?" (2008) 110 R. du N. 520 : "C'est précisément cette inflation des références à la " sécurité juridique " qu’il est souhaitable de comprendre, ce qui, d'un point de vue théorique, impose d'analyser les fonctions que remplissent ces énoncés dans les discours juridiques, et plus particulièrement dans l'argumentation juridique ". 
pratique a pour les participants " 11 . Notre étude a donc pour objet " moins les règles de droit valides et identifiables empiriquement, que le discours, le raisonnement et l'argumentation juridique qui constituent la pratique juridique dans son ensemble "12. Cette étude se situe donc au-delà des sources positives du droit parce qu'elle vise à " rendre compte du point de vue des juristes "13. Les " outils de connaissance " sont donc strictement "textuels " et "documentaire[s]" parce que nous nous intéressons à ce qui "forment un discours sur le droit"14. Notre analyse est

11. Luc B. Tremblay, "La théorie constitutionnelle canadienne et la primauté du droit " (1994) 39 McGill L.J. 101 aux pp. 141-142.

12. Sur cette approche, voir ibid. aux pp. 141-142: "démarche qui, tant en sciences humaines qu'en théorie générale du droit, consiste moins à observer empiriquement les faits qu'à comprendre les pratiques et les actions humaines. Cette approche, qualifiée d'herméneutique, repose sur le postulat que les pratiques sociales, les actions, les comportements, les discours ou autres phénomènes sociaux, possèdent une dimension que ne possèdent pas les faits bruts de la nature. Cette dimension correspond à ce qu'on peut appeler leur "signification interne". Contrairement aux faits bruts de la nature qui n'ont pas conscience de ce qu'ils sont ni de ce qu'ils font, les actions humaines et pratiques sociales ont un sens pour ceux qui les produisent. [...] la compréhension de la signification d'une pratique sociale passe nécessairement par la clarification de son sens interne, c'est-à-dire le sens que cette pratique a pour les participants. [...] Selon ces postulats [méthodologiques qui caractérisent la théorie générale du droit contemporaine] la théorie du droit doit avoir pour objet moins les règles de droit valides et identifiables empiriquement, que le discours, le raisonnement et l'argumentation juridique qui constituent la pratique juridique dans son ensemble ".

13. Devinat, supra note 9 à la p. 10.

14. Ibid. à la p. 10 ; Sur cet aspect, notre méthode est semblable à celle d'un historien. En ce sens : Paul Veynes, Comment on écrit l'histoire. Essai d'épistémologie juridique, Paris, Éditions du Seuil, 1971 aux pp. 274276 : "[D]eux siècles de spéculation historiciste ont trop associé le mot histoire à ceux de science ou de philosophie, alors que le lieu naturel de l'histoire, connaissance documentaire du concret, se place vers le pôle opposé, celui de l'érudition. [...] En somme, ce qui fonde en raison l'érudition est que ni l'histoire ni la poésie ne relèvent d'une intuition immédiate, mais se perçoivent à travers des documents ou des textes. [...] le genre historique, se réduirait à son noyau essentiel et indestructible, l'érudition ". 
Perspectives pour un principe de sécurité juridique

(2009-10) 40 R.D.U.S. en droit canadien :

descriptive, mais "de par la nature de l'objet étudié - le discours sur le droit -, elle prêtera elle-même à discussion "15.

L'objet de notre étude est limité au discours juridique européen et canadien ${ }^{16}$. Elle se limite au discours juridique du 20e et $\mathrm{du} 21^{\mathrm{e}}$ siècle, essentiellement parce que cette période correspond à celle de son émergence. En retour, ce choix ne repose pas sur la prétention qu'il existe "une césure historique " dans la "représentation " 17 de la sécurité juridique tant il est vrai qu'un regard a posteriori sur l'histoire permet de découvrir, bien

15. Michel Boudot, Le dogme de la solution unique. Contribution à une théorie de la doctrine en droit privé, thèse de doctorat, sous la direction de Otto Pfersmann, Faculté de droit et de science politique de l'Université d'AixMarseille, 1999 [non publiée] à la p. 3. Sur cette question, comparer Michel Villey, Leçons d'histoire et de philosophie du droit, Paris, Dalloz, 1962 aux pp. 291-292 : "Le droit n'est pas un objet pur; il n'existe pas, on le fait. Et qu'il en ait ou non conscience, le juriste défend une cause. Décrire pour lui c'est choisir "; Jean-François Gaudreault-Desbiens, "Identitarisation du droit et perspectivisme épistémologique. Quelques jalons pour une saisie juridique complexe de l'identitaire " (2000) 13 Can. J. L. \& Jur. 33 : "[...] la science du droit ne peut prétendre, comme la physique ou la chimie, être froidement descriptive, car les doctrines des juristes se veulent destinées à guider la jurisprudence "; Chritian Atias, Épistémologie juridique, Paris, Presses Universitaires de France, 1985 à la p. 166 : "L'épistémologie démontre aujourd'hui que ce qui est connu n'est jamais totalement indépendant de celui qui connaît, de la façon qu'il a de connaître, de ses convictions et de sa démarche intellectuelle "; Bjarne Melkevik, Horizons de la philosophie du droit, Québec, Presses de l'Université Laval, 1998 à la p. 10 : Les auteurs ne peuvent " faire l'objet d'une recherche de la Vérité " et nous devons les considérer comme "des interlocuteurs avec qui nous confrontons nos idées " grâce au " dialogue "; Mathieu Kessler, Nietzsche ou le dépassement esthétique de la métaphysique, Paris, Presses Universitaires de France, 1999 aux pp. 4, 9 : " [I]l n’y a pas de faits mais seulement des interprétations " et celles-ci sont "lieutenant du sens "; Christophe Grzegorczyk, Françoise Michaut et Michel Troper, Le positivisme juridique, Paris, L.G.D.J., 1992 à la p. 174 : "[...] le positivisme juridique [prétend déposer] les jugements de valeur [...] Il a illusion de le faire; en réalité il prend position, même s'il ne s'en aperçoit pas et croit être objectif. La prétention d'être éthiquement neutre est infondée ".

16. Nous présenterons plus spécifiquement le discours des juristes ayant publié en français.

17. Devinat, supra note 9 à la p. 23. 
avant l'émergence du concept de sécurité juridique au 20e siècle, le souci implicite d'accessibilité, de prévisibilité et de stabilité des normes juridiques dans le discours de certains philosophes ${ }^{18}$ et juristes ${ }^{19}$. "À aucune époque de son histoire, dans aucun système

18. Voir par ex. Thomas Hobbes, Léviathan ou Matière, forme et puissance de l'État chrétien et civil, trad. par Gérard Mairet, Paris, Gallimard, 2000 aux pp. 226, 231 : " [L]e droit consiste en la liberté de faire ou de ne pas faire, alors que la loi détermine et contraint dans un sens ou dans l'autre ". Si aucune loi n'interdit d'accomplir certaines actions, ce n'est pas un "péché " puisque "[t]ant que les lois n'ont pas été faites, on ne peut les connaître "; John Locke, Traité du gouvernement civil, trad. par David Mazel, 2e éd., France, GF Flammarion, 1992 aux pp. 207, 244-245, 252 : " [L]'autorité législative ou suprême, n'a point droit d'agir par des décrets arbitraires, et formés sur-le-champ, mais est tenue de dispenser la justice, et de décider des droits des sujets par les lois publiées et établies et par des juges connus et autorisés. [...] Un pouvoir arbitraire et absolu, et un gouvernement sans lois établies et stables, ne sauraient s'accorder avec les fins de la société et du gouvernement. [...] “ [L]es lois qui concernent les sujets entre eux, étant destinées à régler leurs actions, doivent précéder ces actions-là " ".

19. Voir par ex. Charles Demolombe, Traité de la publication et des effets et de l'application des lois en général, 3e éd., Paris, Auguste Durand et L. Hachette et Cie, 1865 à la p. 10 : "Le but essentiel des lois positives est précisément de prévenir, à cet égard, l'incertitude et l'arbitraire en déterminant, parmi les règles si nombreuses et quelquefois même si controversées du droit naturel, celles qui deviendront lois! Celles qui seront légalement obligatoires "; Jean-Étienne-Marie Portalis, "Discours préliminaire du premier projet de Code civil (1801)" (23 juillet 2008), en ligne : UQAC < http://decouverte.uquebec.ca/primo_library/libweb/actio $\mathrm{n} /$ search.do?mode $=$ Basic\&vid=UQAC\&dscnt=0\&srt=rank\&ct=search\&frb $\mathrm{g}=\& \mathrm{scp} . \mathrm{scps}=\mathrm{scope} \% 3 \mathrm{~A}(\mathrm{CLAA}) \& \mathrm{ind} \mathrm{x}=1 \& \mathrm{dum}=$ true \&dstmp $=12790313657$ $81 \& \mathrm{fn}=$ search\&vl(freeText0)=Discours $\% 20$ preliminaire $\% 20 \mathrm{du} \% 20$ premier \%20projet\%20de\%20Code\%20civil\&vl(119722 14UIO)=any\&tab=default_ta b\&vl(69065447UI1)=all_items\&fromLogin=true $>$ : "[Il] ne faut point de lois inutiles; elles affaibliraient les lois nécessaires; elles compromettraient la certitude et la majesté de la législation [...] [L]es lois ne [peuvent] obliger sans être connues [...Or] les hommes ne peuvent que se "contenter d'une publicité relative, qui, si elle ne peut produire à temps, dans chaque citoyen, la connaissance de la loi à laquelle il doit se conformer, suffit au moins pour prévenir tout arbitraire sur le moment où la loi doit être exécutée ". 
Perspectives pour un principe de sécurité juridique

(2009-10) 40 R.D.U.S. en droit canadien :

les pistes du droit européen

juridique, la sécurité juridique n'est absente des préoccupations sur le droit ${ }^{20}$.

La signification du concept de sécurité juridique est dépendante du contexte juridique, culturel et politique dans lequel il s'inscrit21. La notion de sécurité juridique "a vocation à être interprétée en fonction du système qui la met en œuvre »22. Les juristes européens et canadiens n'appréhendent pas de la même façon le thème de la sécurité juridique et ce concept ne renvoie pas à des réalités de même nature ${ }^{23}$. Ainsi, une analyse séparée de

20. Jean-Louis Bergel, "La sécurité juridique» (2008) 110 R. du N. 271 à la p. 273 [Bergel, "sécurité juridique»]. Pour une perspective historique du concept de sécurité juridique, voir Philippe Raimbault et Dominique J.M. Soulas de Russell, "Nature et racines du principe de sécurité juridique : une mise au point" (2003) 55 R.I.D.C. 85.

21. Maire-Claire Belleau et Rebecca Johnson, "La diversité identitaire et les opinions dissidentes de la Cour suprême du Canada : Conséquences sur la sécurité juridique " (2008) 110 R. du N. 319 à la p. 322; Comparer Jean Grondin, L'universalité de l'herméneutique, Paris, Presses Universitaires de France, 1993 à la p. 182 : selon Gadamer, " comprendre un discours, cela ne se laisse jamais réduire à la saisie intellectuelle d'un état de fait objectif par un sujet, cela relève plus de l'appartenance à une tradition en formation, c'est-à-dire à un dialogue en cours à partir duquel ce qui est dit acquiert sens et cohérence pour nous [...] la compréhension est d'abord affaire de participation, de participation à un sens, à une tradition, ultimement, à un dialogue ".

22. Anne-Laure Valembois, La constitutionnalisation de l'exigence de sécurité juridique en droit français, Thèse de doctorat en droit, sous la direction de Jacques Robert, Université de Panthéon-Assas (Paris II), Paris, L.G.D.J., 2005 à la p. 418 ; Sylvia Calmes, "Le principe de sécurité juridique en droit allemand, communautaire et français ", (2008) 110 R. du N. 287 à la p. 303 : "La sécurité juridique est 'axiomatique', inhérente à la notion même d'ordre juridique, de sorte que son évocation ne revêt finalement de signification particulière que par l'usage que décident d'en faire les acteurs - notamment les juges - d'un système juridique donné ".

23. Comparer Sylvia Calmes, Du principe de protection de la confiance légitime en droit Allemand, Communautaire et Français, t. 1, Paris, Dalloz, 2001 à la p. 113 [Calmes, principe de protection]; Paolo Mengozzi, "Évolution de la méthode suivie par la jurisprudence communautaire en matière de protection de la confiance légitime : De la mise en balance des intérêts, cas par cas, à l'analyse en deux phases ", trad. par L. Duhannoy, Revue du Marché Unique Européen, 1997 à la p. 16. 
Perspectives pour un principe de sécurité juridique

en droit canadien:

(2009-10) 40 R.D.U.S.

les pistes du droit européen

la signification du concept de sécurité juridique dans le discours des juristes européens et canadiens s'impose ${ }^{24}$.

Durant presque la totalité du $20^{\text {e }}$ siècle, la sécurité juridique est considérée dans le discours doctrinal européen comme un "objectif " du droit ou, en d'autres mots, comme une "valeur "vers laquelle les systèmes juridiques doivent tendre. En tant que "valeur", les juristes conçoivent la sécurité juridique comme une norme générale de caractère non juridique. Or, depuis les années 1990, il y a introduction dans le discours de la doctrine européenne et du juge européen d'une conception qui attribue à la sécurité juridique le statut de principe de droit d'où peuvent être déduites des normes juridiques. La sécurité juridique ferait donc, selon cette deuxième conception, partie intégrante du droit positif, source de droit.

Encore aujourd'hui, il y a coexistence dans le discours des juristes de cette double conception de la sécurité juridique. Ainsi, le discours des juristes sur la sécurité juridique ne correspond pas à un système de pensée monolithe ${ }^{25}$. Malgré ce constat, pour des considérations pratiques, nous divisons ces deux conceptions pour mieux les présenter ${ }^{26}$. Nous aborderons donc tour à tour ces

24. Pierre Legrand, "Comparer" (1996) R.I.D.C. à la p. 293 : "Ce n'est, en effet, qu'à travers une analyse des structures cognitives fondant une tradition juridique et permettant d'expliciter les formes de la connaissance juridique qui y sont précisées et qui continuent à régir le processus de fabrication et d'interprétation du droit qu'il est possible au comparatiste d'expliquer comment et pourquoi le droit est ce qu'il est, là où il est. Le comparatiste doit donc chercher à situer le corps complexe des données empiriques qui retiennent son attention dans un contexte socio-cognitif plus large faisant, notamment, sa place à l'historique, au social, au culturel. C'est cette association entre la partie et le tout, l'articulation entre, par exemple, la proposition normative et la culture juridique, qui permet au comparatiste d'affirmer sa compétence spécifique. [...] Lui seul peut donner à la partie le sens dont elle reste autrement apparemment dépourvue sans sa réinsertion dans une totalité ".

25. En ce sens, Boudot, supra note 15 à la p. 9.

26. Évidemment, ce constat de l'existence de deux conceptions n'est pas une négation de la présence de plusieurs autres. 
Perspectives pour un principe de sécurité juridique

(2009-10) 40 R.D.U.S. en droit canadien :

deux conceptions dans le discours juridique, soit la sécurité juridique comme valeur (Section I) et comme principe de droit positif (Section II).

Le discours juridique canadien est caractérisé par un souci implicite de sécurité juridique. En ce sens, les juristes accordent aux valeurs de certitude, prévisibilité et stabilité du droit une grande place dans leur ordre de discours. En retour, ils ne conçoivent pas la sécurité juridique comme un principe ou une règle de droit positif27 (Section III).

\section{Section I : Un débat à l'ombre des règles: la sécurité juridique comme objectif du droit}

Les juristes européens introduisent au $20^{\mathrm{e}}$ siècle le concept de sécurité juridique dans leurs discours ${ }^{28}$. Selon Philippe Raimbault, la notion de sécurité juridique est présente dans de rares occasions dans la doctrine publiciste classique tout en n'étant pas dotée d'une véritable définition ${ }^{29}$. La sécurité juridique n'est alors pas un thème de réflexion en soi chez les juristes ${ }^{30}$.

Les résultats de nos recherches nous portent à croire que l'expression est clairement affirmée et probablement introduite en 1911 dans le discours doctrinal. Dans son œuvre désormais

27. En ce sens, Pierre Larouche, "Table ronde internationale : Constitution et sécurité juridique - Canada " (1999) A.I.J.C. aux pp. 132-133.

28. Philippe Jestaz et Christophe Jamin, La doctrine, Paris, Dalloz, 2004 à la p. 145 .

29. Raimbault, supra note 7 aux pp. 5-6.

30. Ibid. à la p. 6, n. 30. L'auteur énonce : "R. BONNAR s'en sert par exemple pour justifier l'intangibilité des effets individuels des actes juridiques en énonçant que ce dernier principe est "nécessaire pour assurer la sécurité juridique, qui est chose indispensable pour le bon fonctionnement du commerce juridique ", in Précis de droit administratif, LGDJ, Paris, 1940 à la p. 114. Quant à H. Kelsen, il semble en faire un objectif pour l'ordre juridique en considérant que la formulation des normes doit être faite "de telle façon que les équivoques ou ambiguïtés inévitables soient réduites à un minimum, (...) ce qui assurera réellement le plus haut degré de sécurité juridique", dans Théorie pure du droit, trad. par C. Eisenmann, Dalloz, Paris, 1962 à la p. 463 ". 
classique, René Demogue, affirme que le "plus grand des desiderata de la vie juridique et sociale, son moteur central, [c'est] le besoin de sécurité "31. Il rappelle cependant que le "domaine du droit " ne peut réaliser absolument la sécurité du droit parce qu'il s'agit d'un "idéal utopique dans une certaine mesure " 32 , "tout sacrifier à la sécurité, c'est ne viser qu'à un but qu'il est impossible d'atteindre complètement "33. De plus, la sécurité juridique ne doit pas avoir une portée absolue : "le désir de sécurité si considérable qu'il soit n'est pas tout "34. L'auteur utilise habituellement la notion de sécurité, mais nous avons trouvé une référence à l'expression "sécurité juridique " dans son ouvrage ${ }^{35}$. À l'instar de la doctrine publiciste classique, René Demogue avait recours à la notion de sécurité juridique ou à des notions substantiellement similaires pour justifier le recours au formalisme ${ }^{36}$. Également, "la doctrine publiciste française emprunte la formulation d'origine privatiste de "sécurité du commerce juridique" pour regrouper des règles formelles de bon fonctionnement de l'ordre juridique $" 37$.

Quelques années plus tard, en 1936, le juriste anglais Gustave Radbruch prétend que "la sécurité juridique " est un

31. René Demogue, Les notions fondamentales du droit privé, Paris, Librairie nouvelle de droit et de jurisprudence, 1911 à la p. 63.

32. Ibid. à la p. 86.

33. Ibid. à la p. 87.

34. Ibid.

35. Ibid. à la p. 74 .

36. Raimbault, supra note 7 à la p. 36; N.-J. Mazen, L'insécurité juridique inhérente au système juridique, (thèse Dijon), 1979 aux pp. 74-76; Jacques Flour, "Quelques remarques sur l'évolution du formalisme" dans Le droit privé français au milieu du XX siècle, études offertes à $G$. Ripert, t. 1, Paris, L.G.D.J., 1950 aux pp. 93 et ss.

37. Raimbault, supra note 7 à la p. 35, n. 3. L'auteur énonce : "Cette présentation est par exemple utilisée par B. JEANNEAU, Les principes généraux du droit dans la jurisprudence administrative, Sirey, Paris, 1954 à la p. 92 et ss. La même idée est également énoncée, sans aucunement faire allusion à la sécurité juridique, par M. HAURIOU pour qui "les formalités de procédure constituent, avec la détermination précise des compétences, la principale condition de l'ordre et de la modération dans l'exercice du pouvoir administratif ", dans Précis de droit administratif et de droit public, Sirey, Paris, 1927 à la p. 418 ". 
Perspectives pour un principe de sécurité juridique

(2009-10) 40 R.D.U.S. en droit canadien :

les pistes du droit européen

" principe prédominant, [dans] toute l'histoire du droit anglais "38. Il rappelle qu'il y a un conflit inévitable et inéluctable entre la justice, le but du droit et la sécurité. Ces trois éléments s'exigent et se contredisent mutuellement, car " tout renforcement de l'un entraîn[e] un affaiblissement de l'autre "39. Radbruch en conclut que ce serait une grave erreur que de vouloir supprimer cette structure antinomique en donnant la préférence à l'un de ces éléments. Il indique que la véritable méthode consiste plutôt à faire ressortir les antinomies et à les conserver dans une vue d'ensemble 40 .

Le juriste allemand Wilhelm Sauer dans une traduction française de Roubier et Mankiewicz (1937) consacre un article clef sur la sécurité juridique. Il y affirme que "la sécurité juridique entre en conflit avec la justice [...] qui passe pour être le premier des devoirs moraux et sociaux de celui-ci "41. En effet, "[1]a sécurité juridique, au sens de conformité aux normes et d'ordre, s'oppose à la justice et aux considérations d'opportunité supérieures au sens qu'attribue à ces principes l'idée du droit » ${ }^{42}$. En ce sens, pour Sauer, seul le droit juste au point de vue du fond est considéré comme du droit ${ }^{43}$. Par conséquent, lorsqu'une norme juridique particulière produit dans un cas concret un résultat contraire à la justice, le juge doit "utiliser tous les moyens [respectant le formalisme et la sécurité juridique] dont il dispose pour aboutir dans le cas concret à un résultat juste, c'est-à-dire conforme à l'idée du droit "44. Si le respect de la sécurité juridique l'empêche de rendre justice, "le juge doit s'engager dans la voie,

38. Gustav Radbruch, "La sécurité en droit d'après la théorie anglaise " (1936) A.P.D.S.J. à la p. 89.

39. Ibid. à la p. 87.

40. George Gurvitch, "Une philosophie antinomique du droit: Gustave Radbruch ", (1932) A.P.D.S.J. à la p. 537.

41. Wilhelm Sauer, "Sécurité juridique et justice ", trad. par Paul Roubier et Henri Mankiewicz dans Introduction à l'étude du droit comparé Recueil d'Études en l'honneur d'Édouard Lambert, t. 5, Paris, L.G.D.J., 1938 à la p. 34.

42. Ibid. à la p. 39.

43. Ibid. à la p. 37.

44. Ibid. aux pp. 41-42. 
qui est la plus dure et constitue son ultime recours, et justifier devant soi-même et devant les autres la nullité et l'inefficacité de ce droit qui est injuste "45. C'est ainsi, constate Sauer, que l'on parvient à l'harmonie entre la loi et l'idée du droit, entre la sécurité juridique et la justice ${ }^{46}$. Les juristes devraient donc accepter un "système de relativisme " dans la mesure où il peut y avoir selon le contexte et l'époque une prédominance de la sécurité juridique ou de la justice ${ }^{47}$.

Dans le célèbre ouvrage Le déclin du droit (1949) au chapitre intitulé L'insécurité juridique, Georges Ripert exploite habilement la fonction critique du concept : "la servitude est supportée par la certitude de correction qu'elle donne à l'action " et la pire chose est l'arbitraire ${ }^{48}$. Il en conclut que la sécurité juridique est la première valeur que les ordres juridiques doivent atteindre ${ }^{49}$. L'insécurité juridique dont parle Ripert découle notamment du fait que la loi qui donne la règle de conduite n'est pas édictée clairement et précisément50. Selon lui, elle est plus dangereuse que "l'insécurité de fait car elle détruit l'autorité "51. Une procédure de création, d'application et de validité des règles qui est claire et certaine et qui permet d'assurer l'ordre et la connaissance de ses droits constitue l'élément essentiel de la sécurité juridique ${ }^{52}$.

Dans son œuvre, Ripert analyse quelques causes de l'insécurité juridique qu'il considère comme indésirables en droit français. Selon lui, elle est premièrement causée par la difficulté de connaître la loi applicable. Également, nous dit Ripert, l'insécurité naît de l'incertitude de savoir à partir de quel moment une loi doit s'appliquer et jusqu'à quel moment ${ }^{53}$. D'après Ripert, malgré ce constat de la difficulté à connaître la loi "[n]ous

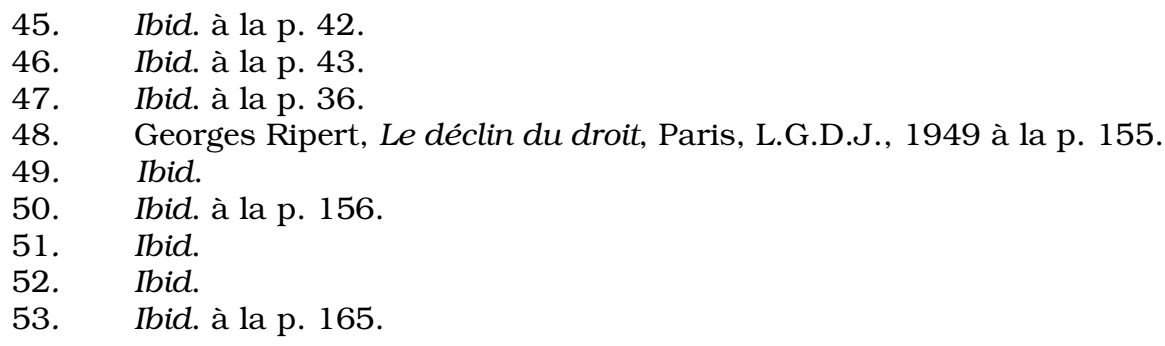


Perspectives pour un principe de sécurité juridique

(2009-10) 40 R.D.U.S. en droit canadien :

les pistes du droit européen

continuons à dire que nul n'est censé ignorer la loi. Mais il faut reconnaître quelque mérite à ceux qui la connaissent "54.

Durant le troisième quart du $\mathrm{XX}^{\mathrm{e}}$ siècle, les professeurs Batiffol et Roubier ont élaboré une doctrine qui considérait la justice, le progrès social et la sécurité juridique comme les valeurs qui inspirent l'ordre juridique ${ }^{55}$. Selon Roubier, "là où cette valeur essentielle qu'est la sécurité juridique a disparu, il n'y a plus aucune autre valeur qui puisse subsister, le mot même de progrès devient dérision, et les pires injustices se multiplient avec le désordre "56. Ainsi, sans sécurité juridique, aucune autre valeur ne peut subsister ${ }^{57}$ puisqu'elle conditionne la justice et le progrès social. Selon Gwenaëlle Dufour, certains ont critiqué les idées de Roubier qui subordonneraient le droit à la positivité en ne tenant pas compte également de l'impératif de justice ${ }^{58}$. Batiffol estime plutôt que " la justice lui importe toujours " parce qu'il " ne lui est pas indifférent que l'ordre s'établisse de n'importe quelle manière "59. Selon Batiffol, " un ordre injuste n'est pas un ordre, mais le désordre "60.

54. Ibid.

55. Voir Henry Batiffol, Problème de base de philosophie du droit, Paris, L.G.D.J., 1979 à la p. 394 [Batiffol, Problème]; Jean-Louis Bergel, Théorie générale du droit, 3e éd., Paris, Dalloz, 1999 à la p. 19; Paul Roubier, Théorie générale du droit, 2e éd., Paris, Sirey, 1951 à la p. 318; Bichara Tabbah, "La trilogie : sécurité, justice et progrès social " dans Théorie générale du droit et droit transitoire. Mélanges en l'honneur de Paul Roubier, t. 1, Paris, Dalloz-Sirey, 1961 à la p. 459.

56. Roubier, ibid. à la p. 334.

57. Paul Roubier, Théorie générale du droit, Paris, Sirey, 1er éd., 1946 à la p. 279.

58. Gwenaëlle Dufour, Sécurité juridique et règle de droit. Illustration en droit des contrats, thèse de doctorat en droit privé, sous la direction de Ch. Jestaz, Université Lille II, 2005 [non publiée] à la p. 9.

59. Henry Batiffol, "La théorie générale du droit de P. Roubier " dans Choix d'articles, Paris, L.G.D.J., 1976 à la p. 354.

60. Batiffol, Problème, supra note 55 à la p. 399. 
Perspectives pour un principe de sécurité juridique

en droit canadien :

(2009-10) 40 R.D.U.S.

les pistes du droit européen

Il appert que les juristes préfèrent inclure la sécurité juridique dans un processus "dialectique "61, en lui préférant certaines valeurs dans certaines circonstances. Encore aujourd'hui, les juristes nuancent l'hégémonie d'une conception absolutiste de la sécurité juridique, l'introduisent dans le cadre de réflexion sur la hiérarchie des valeurs en droit. Par exemple, Perelman observe que la recherche d'un équilibre entre le progrès, la justice et la sécurité juridique constitue l'un des objectifs du droit ${ }^{62}$. Également, Josse Mertens De Wilmars et Jacques Steenbergen considèrent que le droit est une recherche permanente d'équilibre entre "les exigences fondamentales, mais parfois antinomiques, de la liberté et de la justice et les nécessités non moins impérieuses de la sécurité juridique et de l'efficacité, dans la régulation inévitable d'un certain nombre de rapports entre êtres humains "63. Enfin, les juristes opposent souvent à la sécurité juridique le principe de mutabilité du droit essentiel à son évolution ${ }^{64}$.

La sécurité juridique est généralement considérée comme une valeur relative, et décrite comme l'une des raisons d'être du

61. Sur cette question précise, voir Gurvitch, supra note 40 aux pp. 530-563; Henri Radbruch, "Conception dialectique des antinomies juridiques" dans Les antinomies en droit, Bruxelles, Bruylant, 1965 aux pp. 387-388, 391.

62. Chaïm Perelman, Éthique et droit, Éditions de l'Université de Bruxelles, 1990 à la p. 755; Chaïm Perelman, "À propos de l'idée d'un système de droit " dans Le raisonnable et le déraisonnable en droit, Paris, L.G.D.J., 1984 à la p. 98.

63. Josse Mertens de Wilmars et Jacques Steenbergen, "La notion de sécurité juridique dans la jurisprudence de la Cour de justice des Communautés européennes " dans Mélanges offerts à Robert Legros, Éditions de l'Université de Bruxelles, 1985 à la p. 449.

64. Voir notamment Valembois, supra note 3 à la p. 468; Jean Bigot, "Ouverture du colloque " (1998) 3 R.G.D.A. dans Insécurité juridique et assurance à la p. 414; Frédéric Pollaud-Dulian, "À propos de la sécurité juridique" (2001) 3 R.T.D. civ. 488; Paul Fouret, "L'ambiguïté et l'insécurité juridique " (1998) 3 R.G.D.A. dans Insécurité juridique et assurance à la p. 450; Pierre Sargos, "Peut-on parler d'insécurité juridique " (1998) 3 R.G.D.A. dans Insécurité juridique et assurance à la p. 426; François Ost, "Les multiples temps du droit " dans Le droit et le futur, Paris, Presses Universitaires de France, 1985 à la p. 115. 
Perspectives pour un principe de sécurité juridique

(2009-10) 40 R.D.U.S. en droit canadien :

les pistes du droit européen

droit et de la règle de droit. Quel que soit le sens qui lui est attribué, que ce soit à titre de fondement, de justification, d'exigence, de droit naturel, de mission, de fonction, d'essence, de valeur fondamentale du droit ou de l'une de ses finalités ${ }^{65}$, cette conception de la sécurité juridique établie presque toujours un lien direct organique ou symbiotique avec le droit. La notion de sécurité juridique est considérée comme inhérente à la notion de droit66.

On peut comprendre en ce sens que certains juristes considèrent que l'expression "sécurité juridique " serait "tautologique ou redondante "67 même si cette conception est contestée68. "[C]ette présentation dominante de l'exigence première d'une sécurité juridique inhérente au droit [...] a [également] été ponctuellement dénoncée comme procédant d'une illusion rationaliste ${ }^{69}$. Le caractère irréductible ${ }^{70}$ et illusoire ${ }^{71} \mathrm{de}$

65. Pour les nombreuses références, voir Martin Nadeau, Sur les traces d'un principe de sécurité juridique en droit canadien. Les pistes du droit européen, mémoire de maîtrise en droit, sous la direction de Mathieu Devinat, Université de Sherbrooke, 2009 [non publié].

66. Jean-Louis Bergel, Théorie générale du droit, 3e éd., Paris, Dalloz, 1999 à la p. 282 : "la sécurité est liée à l'idée même du droit "; Bergel, "sécurité juridique", supra note 20 à la p. 273 : "l'impératif de sécurité juridique est inhérent au droit et constitue nécessairement l'une des valeurs fondamentales du droit "; Dufour, supra note 58 à la p. 9 : "la sécurité est incontestablement consubstantielle au droit ".

67. Jean Boulouis et Roger-Michel Chevallier, Grands arrêts de la Cour de Justice des Communautés européennes, t.1, 6e éd., Paris, Dalloz, 1994 à la p. 76; Jean Boulouis, "Quelques observations à propos de la sécurité juridique " dans Mélanges Pescatore, 1987 à la p. 53.

68. Dufour, supra note 58 aux pp. 6-7.

69. Sylvie Cimamonti, supra note 6 à la p. 634. Sur cette question, voir : Serge Morard, "La sécurité juridique en droit public français, une illusion conceptuelle ?", (2008) 110 R. du N. 409.

70. Jérôme Kullman, "Les remèdes à l'insécurité juridique» (1998) 3 R.G.D.A. dans Insécurité juridique et assurance aux pp. 492, 496; Salviejo, supra note 5 à la p. 402; Jérémie van Meerbeeck, "Le principe de sécurité juridique dans la jurisprudence communautaire : un principe en quête de sens " (2008) 110 R. du N. 497 à la p. 515 : " Il y a donc une insécurité juridique irréductible ".

71. Michel Fromont, "Le principe de sécurité juridique " A.J.D.A. 1996, n spécial aux pp. 35, 42 : "L’idée que la sécurité juridique est une illusion 
l'insécurité juridique a été constamment rappelé par la doctrine. Pour la plupart, "il n'est pas souhaitable de rechercher une sécurité à tout prix " et "il convient d'admettre une certaine dose d'insécurité juridique ${ }^{72}$.

Dans d'autres contextes, si une insécurité juridique est constatée, peu importe la cause, le caractère souhaitable de la sécurité juridique (souvent indiscuté et postulé) permet de dénoncer ${ }^{73}$ l'insécurité juridique et de $\operatorname{proposer}^{74}$ ou prescrire ${ }^{75}$ des solutions pour enrayer les causes d'insécurité juridique identifiées. Les revendications doctrinales d'un droit transitoire de la règle jurisprudentielle dont certaines défendent la théorie du

n'est pas neuve [...] c'est l'idéal de la sécurité juridique qui doit être nuancée "; Hans Kelsen, Théorie pure du droit, trad. par Henri Thévenaz, 2e éd., Neuchâtel (Suisse), Éditions De La Baconnière, 1988 à la p. 155 : "Quand le sens d'une norme est douteux, il y a selon la doctrine traditionnelle une seule interprétation correcte et une méthode scientifique permettrait de la déterminer dans chaque cas d'espèce. Le droit serait un ordre fixe, réglant de façon non équivoque la conduite des hommes et en particulier celle des tribunaux et des autres organes chargés de l'appliquer. Il garantirait sinon une sécurité économique, du moins une sécurité juridique. Une telle idée est cependant illusoire et la Théorie pure, qui s'adonne à la seule recherche de la vérité, est obligée de détruire cette illusion, malgré ses effets très utiles qu'elle peut avoir dans le domaine de la politique "; Valembois, supra note 3 à la p. 8.

72. Antoine Mazeaud, "La sécurité juridique et les décisions du juge " (2006) 651 Dr. Soc. à la p. 752.

73. Bergel, "sécurité juridique ", supra note 20 à la p. 274 : "L'inflation et l'instabilité législative [...] L'insécurité qui résulte de la jurisprudence [...] sources d'insécurité juridique qui menacent la protection des intérêts individuels et qui sont ressenties par les intéressés comme une véritable injustice. Il faut donc réagir!".

74. Mireille Delmas-Marty, "L'enjeu d'un code pénal (réflexions sur l'inflation des lois pénales en France) " dans Mélanges offerts à Robert Legros, Bruxelles, Éditions de l'Université de Bruxelles, 1985 aux pp. 166, 168 : nous devons "savoir où se tient précisément le mal avant de proposer les remèdes ".

75. Christian Mouly, "Le revirement pour l'avenir " J.C.P. 1994. I. 3776 à la p. 326 : "Les normes ne doivent pas être rétroactives [...] Elle porte atteinte au principe supérieur de sécurité juridique "; Jean-Pierre Camby, "Sécurité juridique et insécurité jurisprudentielle " (2006) 6 R.D.P. à la p. 1512 : "Tout ce qui contribue à des évolutions modulées, et qui assure la sécurité juridique est souhaitable". 
Perspectives pour un principe de sécurité juridique

(2009-10) 40 R.D.U.S. en droit canadien :

les pistes du droit européen

revirement pour l'avenir afin d'éviter ou de diminuer l'effet rétroactif de la règle jurisprudentielle constituent un exemple ${ }^{76}$. Ainsi, l'exposition des causes de l'insécurité juridique, d'une part celles qui sont inéluctables, et d'autre part, celles qui peuvent être enrayées ou diminuées, constitue un préliminaire nécessaire à l'offre de solutions. La sécurité juridique est conçue alors comme un " principe-remède à une donnée structurelle du système "77. La dénonciation de l'insécurité juridique résultant du "désordre normatif constitue ainsi un argument qui permet aux professionnels du droit de s'approprier l'énoncé des solutions au "problème" "78.

Cet usage de la sécurité juridique dans le discours des juristes a amené certains auteurs à affirmer que la thématique de la sécurité juridique est une "rhétorique de l'éviction des solutions possibles " parce que l'on "prétend découvrir une solution " à partir d'une "sophistique qui crée l'illusion que la solution prescrite est fournie par le système juridique lui-même "79. La sécurité juridique est utilisée par certains comme un concept neutre qui permet implicitement de soutenir un discours nettement marqué par la prescription. Par exemple, lorsque le juriste affirme que le respect d'une jurisprudence constante et définitive sur un sujet par la Cour est souhaitable et bon pour la sécurité juridique et donc la seule et unique solution possible (et ce, même si cette solution ancienne est injuste). En ce sens, la sécurité juridique est une sorte de "slogan [...] en vue de justifier

76. Pour les nombreuses références, voir Nadeau, supra note 65 aux pp. 3334.

77. Nicolas Molfessis et R. Libchaber, "Source du droit en droit interne, les “avancées" de la sécurité juridique " (2000) R.T.D. civ. 660.

78. Rachel Vanneuville, "Sécuriser le droit pour mieux gouverner les conduites : les enjeux sociopolitiques de la promotion contemporaine de la sécurité juridique ", Colloque : La gouvernance des sociétés contemporaines au regard des mutations de la normativité, Université Paris XII, Val-de-Marne, 13-15 décembre 2007 [non publié]. Transcription disponible en ligne: Université d'Ottawa $<$ http://biblio.cdp-hrc.uottawa.ca/dbtw-wpd/docs/largotec/vanneuville. pdf $>$.

79. Boudot, supra note 15 à la p. 7 . 
et légitimer, non les solutions décrites, mais la solution prescrite $» 80$.

La sécurité juridique, concept hétéronyme aux multiples statuts (règle de droit positif, valeur éthique ou philosophique, objet d'étude) est évidemment un terrain fertile à la sophistique. Or, cet usage ne fait pas exception. En effet, aucun concept juridique n'est exempt de la nécessité d'être interprété selon la précompréhension de l'interprète et aucun concept ne peut contenir en soi un sens fixe et définitif. Toute discussion abstraite et théorique et tout jugement comprennent leur part de subjectivité et de rhétorique. En général, "les principes juridiques sont abondamment mis à contribution pour ne rien exprimer, asservis à la rhétorique " 81 . Les juristes en appellent aux principes pour soutenir leurs pensées, mais en réalité ils ne recherchent quelquefois que des "vêtements magnifiques pour des opinions discutables $" 82$.

La sécurité juridique conçue par la doctrine comme une valeur vers laquelle la science du droit et les systèmes juridiques doivent tendre a donc "une dimension quasi philosophique relevant de l'idéal juridique " 83 . À titre de "valeur "métajuridique", du domaine de l'axiologie " 84 elle est un idéal chargé de l'efficacité symbolique d'un mythe. Contre l'insécurité, la doctrine juridique a recours à son " pouvoir incantatoire $" 85$.

La sécurité juridique fait partie intégrante des grands débats et discussions de la théorie du droit. En particulier, elle participe aux réflexions sur le "droit contemporain, tant dans son rôle social que dans son mode de production "86. Elle est utilisée

80. Ibid.

81. Patrick Morvan, "La sécurité juridique: l'antidote au poison de l'insécurité " (2006) 651 Dr. Soc. à la p. 708.

82. Georges Ripert, Les forces créatrices du droit, 2e éd., Paris, L.G.D.J., 1955 au $\mathrm{n}^{\circ} 132$.

83. Salviejo, supra note 5 à la p. 209.

84. Ibid. à la p. 8.

85. Valembois, supra note 3 à la p. 10.

86. Vanneuville, supra note 78 à la p. 6. 
Perspectives pour un principe de sécurité juridique

(2009-10) 40 R.D.U.S. en droit canadien :

par la doctrine "pour mieux décrire, comprendre et expliquer "87, notamment sur "une base de réflexion critique (au sens constructif du terme) sur les structures de l'ordre juridique " 88 .

\section{Section II : Émergence du principe de sécurité juridique dans le discours juridique européen}

La volonté chez les auteurs européens de faire de la sécurité juridique un véritable principe positif, une norme réelle et non simplement souhaitée, émerge vers la fin des années 1990. Selon cette nouvelle conception, la sécurité juridique est une norme générale juridiquement obligatoire, de laquelle peuvent être déduites d'autres normes. Elle est donc conçue comme un "principe du droit positif " qui protège et concrétise la sécurité juridique. Sous cette forme, il s'agit d'un principe fédérateur de règle ${ }^{89}$, un "sur-principe" ou une "super-notion" ${ }^{90}$. En ce sens, le principe de sécurité juridique est une "règle pour les règles de droit, qui, d'un point de vue technique, doivent répondre à certaines exigences ${ }^{91}$.

Selon cette nouvelle conception qui accorde à la sécurité juridique le statut de "principe de droit positif", celui-ci "n'acquiert un sens et un contenu véritables que parce qu'il se "rattache" à des principes existants qui viennent le soutenir "92. La sécurité juridique est donc considérée, dans le discours de la doctrine européenne, comme un principe composé de plusieurs normes juridiques non écrites, mais obligatoires envers les branches de l'État et les sujets de droit.

87. Valembois, supra note 3 à la p. 393.

88. Ibid. à la p. 467.

89. Bertrand Mathieu, "Pour une reconnaissance de principes matriciels en matière de protection constitutionnelle des droits de l'homme " D. 1995. Chron. 211; Bertrand Mathieu, "Table ronde internationale : Constitution et sécurité juridique - France " A.I.J.C. 1999 à la p. 155.

90. Willy Zimmer, "Table ronde internationale: Constitution et sécurité juridique - Allemagne " A.I.J.C. 1999 à la p. 91.

91. Dufour, supra note 58 à la p. 12.

92. Ibid. à la p. 23. En ce sens voir Valembois, supra note 3 à la p. 13. Voir aussi Zimmer, supra note 90 à la p. 91. 
Dans cette perspective très orientée vers la technique du droit, le principe de sécurité juridique a une "fonction de sécurisation du système juridique "93. Ce principe de sécurité juridique élaboré par la doctrine récente vise, en effet, à rendre conforme aux exigences de la sécurité juridique "la règle de droit, soit-elle de nature législative, réglementaire ou jurisprudentielle ${ }^{94}$.

Plusieurs auteurs qui attribuent le statut de règle de droit à la sécurité juridique considèrent la sécurité juridique comme "inhérente au modèle théorique de l'État de droit "95 et comme une " exigence liée à l'État de droit "96. Pour certains, si le principe de l'État de droit est expressément consacré en droit constitutionnel d'un pays, le principe de la sécurité juridique déduit du principe de l'État de droit en tire donc sa valeur constitutionnelle, sa source juridique et est donc implicitement consacré en droit positif 97 . Certains vont même jusqu'à affirmer que la sécurité juridique est un principe positif obligatoire pour tous les systèmes de droit puisqu'elle est inhérente au principe de l'État de droit lui-même universel ${ }^{98}$.

93. Valembois, supra note 3 à la p. 8.

94. Michèle de Salvia, "La place de la notion de sécurité juridique dans la jurisprudence de la Cour européenne des droits de l'homme " C.C.C. 200, $\mathrm{n}^{\circ} 11$ à la p. 94.

95. Valembois, supra note 3 à la p. 29.

96. Ibid. à la p. 28.

97. Salviejo, supra note 5 à la p. 17: sécurité juridique est l'un des fondements de l'État de droit, ce qui permet d'affirmer qu'elle possède "des bases textuelles nationales, souvent constitutionnelles "; Dufour, supra note 58 à la p. 472 : "En effet, malgré l'absence de valeur juridique formelle de la sécurité juridique, celle-ci a une réalité dans le système juridique français. En tant qu'impératif rattaché à l'État de droit, la sécurité juridique prescrit des règles relatives à l'élaboration du droit positif. C'est une règle des règles de droit, une métarègle ".

98. Calmes, principe de protection, supra note 23 à la p. 111 : la sécurité juridique est un véritable principe du droit parce qu'elle est "une nécessité apparente à tout ordre juridique " en tant que "composante élémentaire de l'État de droit ". 
Perspectives pour un principe de sécurité juridique

(2009-10) 40 R.D.U.S. en droit canadien :

Voici un exemple de ce type de raisonnement. En parlant du droit communautaire, la juriste Caroline Salviejo suggère que "si la sécurité juridique ne fait pas l'objet d'une inscription explicite en ces termes dans le corps de ses dispositions conventionnelles, on ne peut considérer qu'elle y est absente dans la mesure où elle est, normalement, inhérente à tout système de droit qui doit répondre à des critères de stabilité, de prévisibilité et d'accessibilité "99. Elle conclut donc que le principe de sécurité juridique "dont la qualification est absente, trouve ses racines dans le principe de prééminence du droit, principe fondamental d'interprétation majeure dans l'œuvre du juge européen "100. Michèle de Salvia utilise le même raisonnement pour ce qui est du droit de la Convention européenne des droits de l'homme : "la sécurité juridique du justiciable est précisément un des objectifs de la $\mathrm{CEDH}$, car il se rattache directement à un des principes généraux d'interprétation auquel la jurisprudence de Strasbourg se réfère fréquemment et qui est d'ailleurs proclamé dans le Préambule de la CEDH : la prééminence du droit "101.

Dans d'autres contextes, la doctrine ne prétend pas que le principe de sécurité juridique tire sa source du principe de l'État de droit, mais s'efforce à montrer combien les préoccupations sécuritaires étayent depuis longtemps les décisions des tribunaux supérieurs, en quoi la sécurité juridique dans le " non-dit " est une "réalité sur tous les fronts jurisprudentiels" et que les Cours, "[s]ans affirmer la notion, [...] en garanti[es] progressivement les composantes "102. Il s'agirait d'une véritable stratégie doctrinale qui vise la promotion de la sécurité juridique. Ainsi, "certaines études doctrinales [...] invitent à considérer que l'exigence de sécurité juridique a matériellement une valeur constitutionnelle et

99. Salviejo, supra note 5 à la p. 4.

100. Ibid. à la p. 46.

101. De Salvia, supra note 94 à la p. 94.

102. Jean-Pierre Camby, "La sécurité juridique : une exigence juridictionnelle " (2006) R.D.P. à la p. 1172. 
qu'elle est un outil implicitement actionné par le juge constitutionnel français »103.

Sous l'influence du droit communautaire, certains prescrivent simplement sa consécration en droit positif interne ${ }^{104}$, notamment parce que les atteintes croissantes à la sécurité juridique justifient d'élever celle-ci en principe ${ }^{105}$. Enfin, certains auteurs contestent l'idée que la sécurité juridique est un principe ou remettent en question l'opportunité de sa consécration en droit positif 106 .

Cette nouvelle conception de la sécurité juridique comme un principe de droit dans la doctrine européenne n'est certainement pas étrangère au fait que certaines juridictions nationales et supranationales européennes accordent à la sécurité juridique le prestigieux statut de principe de droit positif.

103. Valembois, supra note 3 à la p. 460; Voir par ex. Bertrand Mathieu, "La sécurité juridique: un principe constitutionnel clandestin, mais efficient" dans Mélanges Patrice Gélard, Paris, L.G.D.J., 2000 à la p. 302.

104. Emma Ben Merzouk, "La fausse consécration du "principe de sécurité juridique" "D. 2003. Jur. à la p. 2723 : "Certains aspirent, [...] à la consécration d'un 'principe de sécurité juridique' "; Morvan, supra note 81 à la p. 715 : "La juridiction du Palais-Royal pouvait-elle résister encore longtemps sur la pente naturelle conduisant à la diffusion des principes généraux du droit communautaire au sein du droit français? La doctrine administrative l'y invitait".

105. Molfessis et R. Libchaber, supra note 77 à la p. 660.

106. Calmes, thèse, supra note 2 à la p. 117 : la sécurité juridique devrait " plutôt rester une ligne directrice concrétisée par des exigences précises, une perspective, un souci, tout au plus un impératif "; Antoine Cristau, "L'exigence de sécurité juridique» D. 2002.chron.2816; Bernard Pacteau, "La sécurité juridique, un principe qui nous manque?" A.J.D.A. 1995, n spécial aux pp. 153, 156 : " on ne peut que préférer à l'émergence d'un principe de sécurité juridique ce qu'on appellerait plutôt l'instillation dans notre droit d'une perspective de sécurité [...] Le thème de la sécurité juridique est plus riche en inspiration qu'il ne peut l'être en implications directes "; Emma Ben Merzouk, Le principe de sécurité juridique en droit positif, thèse de doctorat en droit, Université Paris II, 2003 [non publiée] : L'objet de cette thèse est de montrer l'absence de nécessité d'une consécration par le droit français d'un principe de sécurité juridique. 
Perspectives pour un principe de sécurité juridique

(2009-10) 40 R.D.U.S. en droit canadien :

les pistes du droit européen

Selon certaines études, les juges d'Allemagne107, de Suisse 108 et des Pays-Bas ${ }^{109}$ ont fait application du principe de sécurité juridique et de ses équivalents. Le droit espagnol aurait consacré explicitement l'équivalent d'un principe de sécurité juridique dans leur constitution nationale ${ }^{110}$.

Le principe de sécurité juridique a également fait son introduction en France dans les dispositifs de certaines décisions de la Cour de cassation et du Conseil d'État ${ }^{111}$.

Le juge européen a consacré la sécurité juridique à titre de principe fondamental du droit européen à partir des années 1960.

107. Calmes, thèse, supra note 2 à la p. 11 ; Salviejo, supra note 5 aux pp. 2-3.

108. Fromont, supra note 71 à la p. 178; Calmes, thèse, supra note 2 à la p. 9.

109. Manuel Delamarre, "La sécurité juridique et le juge administratif français " A.J.D.A. 2004, 186.

110. Ibid. Salviejo, supra note 5 à la p. 22; Dufour, supra note 58 à la p. 14; Dominique Colas, Textes constitutionnels français et étrangers, textes essentiels, Paris, Larousse, 1994 à la p. 335 : Article 9 alinéa 3 du texte fondamental espagnol de 1978 énonce que " la Constitution garantit le principe de la légalité, la hiérarchie des normes, leur publicité, la nonrétroactivité des dispositions punitives qui ne favorisent pas ou restreignent des droits individuels, la sécurité juridique et la responsabilité des pouvoirs publics et protège contre toute action arbitraire de ceux-ci ".

111. Voir par ex. Cass. Soc., 2 mai 2000, Bull. civ. 2000. V. 127, n 162 , D. 2000. IR. 168 : Une disposition permettant à l'employeur de modifier unilatéralement le contrat de travail des salariés "suscite une difficulté sérieuse quant à sa légalité au regard du principe de la sécurité juridique "; Cons. d'État, 24 mars 2006, Société KPMG (Assemblée), A.J.D.A. 2006288460 à la p. 841 : "Considérant que le principe de confiance légitime, qui fait partie des principes généraux du droit communautaire, ne trouve à s'appliquer dans l'ordre juridique national que dans le cas où la situation dont on a à connaître le juge administratif français est régie par le droit communautaire [...] Considérant qu'indépendamment du respect de cette exigence, il incombe à l'autorité investie du pouvoir réglementaire d'édicter, pour des motifs de sécurité juridique, les mesures transitoires qu'implique, s'il y a lieu, une réglementation nouvelle; qu'il en va ainsi en particulier lorsque les règles nouvelles sont susceptibles de porter une atteinte excessive à des situations contractuelles en cours qui ont été légalement nouées ". 
Ainsi, le droit européen, à travers le droit de la Cour européenne des droits de l'homme $(\mathrm{CEDH})$ et de la Cour de justice de la Communauté européenne (CJCE), a progressivement élaboré un principe de sécurité juridique.

Le principe de sécurité juridique "prend essentiellement la forme d'une norme jurisprudentielle souple et évolutive " dont la source dans les traités du droit européen " ne représente qu'un lointain support "112. En effet, la Convention européenne des droits de l'homme et les Traités constitutifs des Communautés européennes ${ }^{113}$ ne reconnaissent pas expressément un principe de sécurité juridique.

La CJCE a reconnu dans les années 1960 le principe de sécurité juridique. La CJCE parle alors vaguement du "principe du respect de la sécurité juridique "114. Un an plus tard, la CJCE déclare que "le principe général de sécurité juridique " est " une règle de droit à respecter dans l'application du traité "115. La CJCE a qualifié la sécurité juridique d'impératif 116 d'exigence fondamentale ${ }^{117}$, de règle de droit supérieure ${ }^{118}$, de principe

112. Bertrand Mathieu, "Réflexions en guise de conclusion sur le principe de sécurité juridique "C.C.C. $2001, \mathrm{n}^{\circ} 11$ à la p. 110.

113. Salviejo, supra note 5 à la p. 36 : le principe de sécurité juridique " est formellement absent dans les traités constitutifs des Communautés européennes "; Jürgen Schwarze, Droit administratif européen, Le principe de sécurité juridique, t. II, Bruxelles, Bruylant, 1994 à la p. 207 : la CJCE ne fait référence à aucune source textuelle qui permettrait de "reconnaître l'existence de la sécurité juridique et d'en délimiter le contenu".

114. C.J.C.E., 22 mars 1961, Société nouvelle des usines de Pontlieue Aciéries du Temple (S.N.U.P.A.T.) c/ Haute Autorité de la Communauté européenne $d u$ charbon et de l'acier (C.E.C.A.), aff. 42 et 49/59, Rec.1961.103 [Aciéries du Temple]; Voir aussi C.J.C.E., 13 juillet 1961, Meroni c/ Haute Autorité de la CECA, aff. 14, 16, 17, 20, 24, 26, 27/60 et 1/61, Rec.1961.319.

115. C.J.C.E., 6 avril 1962, De Geus en Uitdenbogerd c/ Bosch, aff. 13/61, Rec. 1962.115.

116. C.J.C.E., 15 décembre 1987, Pays-Bas c/ Commission, aff. 326/86, Rec. 1987.5091.

117. C.J.C.E., 14 juillet 1972, Azianda Colori Nazionali c/ Commission, aff. 57/69, Rec.1972.933 : "l'exigence fondamentale de la sécurité juridique 
Perspectives pour un principe de sécurité juridique

(2009-10) 40 R.D.U.S. en droit canadien :

les pistes du droit européen

fondamental de la Communauté 119 , de principe fondamental120 et enfin de principe général inhérent à l’ordre juridique communautaire ${ }^{121}$.

La première référence explicite et directe du principe de sécurité juridique dans la jurisprudence de la CEDH remonte à 1979 dans l'arrêt Marckx. Le juge européen y indique que "le principe de sécurité juridique, nécessairement inhérent aux droits de la Convention comme au droit communautaire, dispense l'État belge de remettre en cause des actes ou situations juridiques antérieurs au prononcé du présent arrêt "122. Vingt ans plus tard, la $\mathrm{CEDH}$ réaffirmait que la sécurité juridique est un "principe général implicite dans la Convention et [...] constitue l'un des éléments fondamentaux de l'État de droit "123. Notons que "les cas d'application directe de la notion de sécurité juridique sont peu nombreux " 124 , mais existent tout de même. Le principe de sécurité juridique est une sorte d'impératif prise en compte par le juge de la CEDH dans ses motifs. À titre d'exemple, dans l'affaire Cossey,

s'oppose à ce que la Commission puisse retarder indéfiniment l'exercice de son pouvoir d'infliger des amendes ".

118. C.J.C.E., 14 mai 1975, Comptoir national technique et agricole (C.N.T.A.) c/ Commission, aff. 74/74, Rec. 1975553.

119. Calmes, thèse, supra note 2 à la p. 25; Voir aussi Aciéries du Temple, supra note 114 à la p.125: "principes fondamentaux d'une bonne administration et de la sécurité juridique ".

120. C.J.C.E., 16 décembre 1976, Comet BV c/ Produktshap voor Siergewassen, aff. 45/76, Rec.1976.2043 : "la fixation de tels délais, en ce qui concerne les recours de nature fiscale, constitue l'application du principe fondamental de sécurité juridique qui protège à la fois le contribuable et l'administration concernée "; C.J.C.E., 25 janvier 1979, Racke c/ Hauptzollamt Mainz, aff. 98/78, Rec.1979.69 : " un principe fondamental dans l'ordre juridique communautaire exige qu'un acte émanant des pouvoirs publics ne soit pas opposable aux justiciables avant qu'il n'existe pour ceux-ci la possibilité d'en prendre connaissance".

121. C.J.C.E., 27 mars 1980, Administrazione delle finanze dello Stato c/ Denkavit italiana, aff. 61/79, Rec. 19801205.

122. Marckx c./ Belgique (1979), C.E.D.H. Notice A31 à la p. 317.

123. Baranowski c/ Pologne, n²8358/95, [2000] 2000-III C.E.D.H. au par. 58.

124. De Salvia, supra note 94 à la p. 95. 
la $\mathrm{CEDH}$ considère que ses décisions antérieures ne la lient pas "si des raisons impérieuses lui paraissaient le demander "125. Elle rappelle toutefois que, en général, elle " a coutume d'en suivre et [d'en] appliquer les enseignements, dans l'intérêt de la sécurité juridique et du développement cohérent de la jurisprudence relative à la convention »126.

Cette présentation montre que dorénavant certains auteurs et juges partagent ce que certains pourraient décrire comme une croyance collective : la sécurité juridique n'est plus uniquement une valeur, elle constitue dans plusieurs pays européens et en droit supranational européen une norme juridique.

\section{Section III : Émergence dans le discours juridique canadien du concept de sécurité juridique}

Au Canada, les questions relatives à la sécurité juridique se présentent différemment. Nos recherches nous portent à croire que pendant longtemps, le concept de sécurité juridique a été exempt du discours juridique canadien. Or, depuis une vingtaine d'années, la littérature juridique a multiplié les références à cette notion et la sécurité juridique a fait depuis l'objet d'études ponctuelles ${ }^{127}$. Par exemple, l'Université de Sherbrooke a

125. Cossey c/ Royaume-Uni (1990), C.E.D.H. Notice A184 à la p. 35.

126. Ibid.

127. Les articles de doctrine consacrés expressément à l'étude de la sécurité juridique en droit canadien que nous avons trouvés, sont les suivant: Charlotte Lemieux, "Jurisprudence et sécurité juridique : une perspective civiliste " dans Droit contemporain, Cowansville, Yvon Blais, 1998 à la p. 503; Larouche, supra note 27 à la p. 131; Leonard I. Rotman, "The fiduciary concept and the subjective nature of legal certainty " (2008) 110 R. du N. 359; Belleau et Johnson, supra note 21; Jean-Luc Bilodeau, "La sécurité juridique et l'efficience des marchés : le cas de l'AMF " (2008) 110 R. du N. 429; Élise Charpentier, "Le prix de la sécurité juridique en matière contractuelle " (2008) 110 R. du N. 545; Pierre-André Côté, "Le souci de la sécurité juridique dans l'interprétation de la loi au Canada " (2008) 110 R. du N. 685 [Côté, "souci de la sécurité»]; Anne-Françoise Debruche, Mathieu Devinat et Anne Saris, "Préface - Actes du Xe Congrès de l'Association internationale de méthodologie juridique: Sécurité juridique/Legal certainty " (2008) 110 R. du N. 265 : Au Canada, 
Perspectives pour un principe de sécurité juridique

(2009-10) 40 R.D.U.S. en droit canadien :

récemment été l'hôte, en 2007, du Xe Congrès de l'Association internationale de méthodologie juridique, qui a été entièrement consacré à l'étude du concept de sécurité juridique et où plusieurs intervenants canadiens ont participé ${ }^{128}$. Quant aux juges de la Cour suprême, ils utilisent cette expression depuis les années 1990, mais sans vraiment la définir. Cependant, il appert que le droit canadien n'a pas encore attribué de statut formel au principe de sécurité juridique. L'absence d'un principe de sécurité juridique consacré formellement et officiellement dans le discours juridique n'implique cependant pas que le droit canadien ne consacre pas de règles qui protègent la sécurité juridique ${ }^{129}$.

\section{A. Le discours doctrinal}

Pour les auteurs qui évoquent la notion de sécurité juridique, celle-ci est habituellement considérée comme souhaitable. Par exemple, pour une auteure, celle-ci serait une "pierre angulaire du droit, son pilier essentiel "130 et pour un autre, elle constitue un " avantage "131.

Certains auteurs ne définissent pas la notion de sécurité juridique lorsqu'ils en font usage ${ }^{132}$ ou lui attribuent un sens abstrait sans référence ${ }^{133}$. Cependant, lorsqu'ils le font, la majorité des auteurs tirent les éléments de la définition du concept de sécurité juridique à partir de propositions élaborées par la doctrine européenne. Par exemple, lorsque Danielle Grenier définit

la sécurité juridique a "suscité peu d'études approfondies dans les communautés juridiques de common law et de droit civil ".

128. Anne-Françoise Debruche, Mathieu Devinat et Anne Saris, Comité organisateur, Actes du Congrès de l'Association internationale de méthodologie juridique, "Sécurité juridique/legal certainty", Sherbrooke, les 24-27 octobre 2007, (2008) 110 R. du N. aux pp. 265-727.

129. Sur cette question, voir Nadeau, supra note 65 aux pp. 73-92.

130. Catherine Kessedjian, "Le temps du droit au XXI ${ }^{\mathrm{e}}$ siècle - Compatibilité avec la codification?" (2005) 46 C. de D. 547 à la p. 554.

131. Guy Lefebvre et Juliette d'Hollander, "La normalisation des contrats internationaux d'ingénierie " (1997) 31 R.J.T. 209 à la p. 230.

132. Voir par ex. Bilodeau, supra note 127.

133. Voir par ex. Côté, "souci de la sécurité", supra note 127; Larouche, supra note 27 à la p. 131; Charpentier, supra note 127 . 
le concept de sécurité juridique, elle mentionne deux propositions des auteurs Michel Fromont et Moncef Kdhir ${ }^{134}$. Marie-Claire Belleau et Rebecca Johnson évoquent également des auteurs européens ${ }^{135}$ lorsqu'elles définissent la sécurité juridique, soit Gérard Cornu et Moncef Kdhir ${ }^{136}$. Enfin, Charlotte Lemieux évoque un auteur européen lorsqu'elle définit la notion de sécurité juridique, soit D. Foussard ${ }^{137}$.

Cet usage rend compte de l'influence du droit européen en droit canadien. Le sens accordé par les juristes canadiens au concept semble commun à celui développé par la communauté juridique européenne. En effet, l'accessibilité138 (accès formel et intelligibilité du texte), la prévisibilité139 et la stabilité140 du droit recoupent l'ensemble des définitions que l'on a pu dégager de la littérature canadienne ${ }^{141}$. Le dialogue qui s'établit avec les juristes européens permet aux juristes canadiens de s'inspirer de ce qui se fait ailleurs et contribue au renouvellement de la réflexion relativement aux concepts, idéaux et normes juridiques reliés à la sécurité juridique.

134. Danielle Grenier, "La notion de confiance légitime et la jurisprudence des tribunaux de droit administratif " dans Droit contemporain, Cowansville, Yvon Blais, 1998 à la p. 487; Fromont, supra note 71 à la p. 178; Moncef Kdhir, "Vers la fin de la sécurité juridique en droit français? " (1993) 46 Rev. admin. à la p. 538.

135. Belleau et Johnson, supra note 21 aux pp. 322-323.

136. Gérard Cornu, dir., Vocabulaire juridique, $7^{\mathrm{e}}$ éd., Paris, Presses Universitaires de France, 1998 aux pp. 776-777; Moncef Kdhir, supra note 134 à la p. 538.

137. Lemieux, supra note 127 à la p. 505; D. Foussard, "Rapport de synthèse " dans Sécurité du droit et jurisprudence, Entretiens de Nanterre, J.C.P. éd. Entreprise 1990 à la p. 17.

138. Carmen Lavallée, "À la frontière de l'éthique et du droit " (1993) 24 R.D.U.S. 1 aux pp. 20, 22.

139. Lemieux, supra note 127 à la p. 505; Côté, "souci de la sécurité», supra note 127 à la p. 687.

140. Charpentier, supra note 127 à la p. 547; Grenier, supra note 134 .

141. Belleau et Johnson, supra note 21 aux pp. 323-324: "En ce sens, la finalité de la sécurité juridique consiste en 'la recherche d'un système plus stable, accessible et prévisible' ". 
Perspectives pour un principe de sécurité juridique

(2009-10) 40 R.D.U.S. en droit canadien :

les pistes du droit européen

La doctrine canadienne partage avec son homologue européen un discours qui rejette et dénonce une conception trop absolue de la sécurité juridique ${ }^{142}$ qui serait susceptible de justifier l'injustice ${ }^{143}$ ou d'occulter les questions juridiques fondamentales ${ }^{144}$.

142. Voir par ex. Belleau et Johnson, supra note 21 aux pp. 325, 334; Luc B. Tremblay, "Le normatif et le descriptif en théorie du droit " (2002-2003) 33 R.D.U.S. 69 aux pp. 92-93.

143. Voir par ex. Richard Tremblay, L'essentiel de l'interprétation des lois, Cowansville, Yvon Blais, 2004 aux pp. 20-21 : "Une approche aussi pragmatique s'écarte évidemment d'une philosophie positiviste extrême qui recherche avant tout la prévisibilité des jugements, prévisibilité qui serait, selon ses promoteurs, garante d'une plus grande sécurité juridique. Or, on voit mal comment la mise en application d'une norme dont le sens littéral est clair, mais absurde pourrait être gage de sécurité juridique, puisqu'elle ne respecte pas les faits et a toutes les chances d'être source d'injustice "; Jeanne Simard, "L'interprétation législative au Canada : la théorie à l'épreuve de la pratique " (2001) 35 R.J.T. 549 à la p. 554 : "Cette conception de l'interprétation législative, en apparence rassurante pour le milieu juridique canadien, favorise la prévisibilité des décisions de justice et assure une certaine forme de sécurité dans les relations juridiques. [...] La doctrine, pour sa part, propose de plus en plus des modèles faisant appel à des valeurs plus dynamiques que celles de la stabilité des lois et de la sécurité juridique sans pour autant négliger le texte et ses différents contextes d'utilisation ".

144. Jean-François Gaudreault-Desbiens, "Identitarisation du droit et perspectivisme épistémologique. Quelques jalons pour une saisie juridique complexe de l'identitaire " (2000) 13 Can. J.L. \& Jur. 33 aux pp. 71-74: "Certes peut-on souhaiter une réintroduction du doute dans le procès de connaissance juridique [...] Pour bon nombre de juristes, toutefois, pareille démarche ne va pas de soi tant l'idéal positiviste de la sécurité juridique est souvent compris comme une injonction d'adhérer au dogme de l'immobilisme. En fait, la réceptivité des juristes, qu'ils soient universitaires ou praticiens, à l'idée d'un nécessaire questionnement des postulats de leur discipline, de leurs méthodes d'appréhension de certains types de faits ainsi que de l'impact de ces postulats et méthodes sur la pensée juridique, risque en bout de ligne d'être fonction de leur conception de ce qu'est le droit, de ce que doit être sa place dans la société et, surtout, de l'image qu'ils se font de leur propre rôle en tant que juristes. [...] Nous ne prônons pas l'abandon complet des idéaux de sécurité juridique ou de cohérence normative, bien au contraire. Nous prônons strictement leur abandon lorsqu'ils empêchent le questionnement juridique fondamental que nous appelons de nos voeux. Nous souhaitons en fait par là un retour à ce qui nous 
La valeur de sécurité juridique est utilisée par la doctrine comme un concept descriptif (parfois postulé comme souhaitable ou cela se déduit implicitement). Notamment, ce concept lui permet d'identifier les phénomènes constitutifs d'insécurité juridique. Par exemple, c'est ce que fait le professeur Jean-Luc Bilodeau lorsqu'il affirme que "les normes composant le droit québécois des valeurs mobilières connaissent une croissance faramineuse en quantité, en volume et en complexité " et que "ce phénomène nuit à la sécurité juridique des citoyens "145. La professeure Charlotte Lemieux utilise la même approche en relevant les "facteurs" constitutifs de sécurité ou d'insécurité juridique ${ }^{46}$. Par exemple, affirme-t-elle, "[1]a rétroactivité d'une règle de droit est certainement la pire menace pour la sécurité et la prévisibilité du droit. Or, la loi interprétative offre au législateur la malheureuse possibilité de déguiser une rétroactivité substantielle en loi interprétative "147. Également, elle constate "l'abondance des lois rétroactives au Québec, abondance contre laquelle personne ne semble s'insurger, la loi n'est même plus en mesure d'offrir au citoyen la certitude qu'il vit sous l'empire de règles présentes et non sous celles de règles futures, dont la teneur est forcément occulte »148.

Le concept de sécurité juridique est également envisagé selon une deuxième approche en ce qu'il serait un souci implicite ${ }^{149}$ ou une valeur qui sous-tend ou regroupe un ensemble

paraît être le fondement même de ces idéaux, soit la protection des justiciables par le truchement de normes substantielles et procédurales qui sont relativement précises, prévisibles, publiques, mais aussi perçues comme légitimes".

145. Bilodeau, supra note 127 à la p. 431.

146. Lemieux, supra note 127 à la p. 506 : "Nous nous proposons d'examiner la sécurité juridique que l'activité des tribunaux de droit civil est en mesure d'offrir au citoyen [...] Or, en droit civil, certains facteurs nous rapprochent du principe de sécurité juridique et certains facteurs, parfois les mêmes, mais vus sous un angle différent, nous en éloignent ".

147. Ibid. à la p. 513.

148. Ibid. à la p. 511.

149. En ce sens : Pierre-André Côté, Interprétation des lois, 3e éd., Montréal, Thémis, 1999 aux pp. 9, 12, 13, 513; Louise Rolland, "Les Principes 
Perspectives pour un principe de sécurité juridique

(2009-10) 40 R.D.U.S. en droit canadien :

de règles de droit positif ou d'interprétation. Sous cette forme, la sécurité juridique est considérée comme une valeur qui inspire150 et assure le fondement ${ }^{151}$ de certaines méthodes d'interprétation ou de règles de droit positif.

En ce sens, Pierre-André Côté affirme qu'il ne fait "aucun doute que la sécurité juridique soit une préoccupation constante et parfois même déterminante dans l'établissement du sens des lois, que ce soit par le juge ou par les autres acteurs de la vie juridique au Canada "152. Élise Charpentier estime que "le sort réservé aux contrats lésionnaires en droit québécois constitue une illustration particulièrement éloquente de la diversité des règles auxquelles la sécurité juridique a donné naissance au fil du temps "153. Pierre Larouche considère que la sécurité juridique est " un principe inconnu de l'ordre juridique canadien", mais que "plusieurs principes à valeur constitutionnelle (non-rétroactivité des lois, principe de précision ainsi que primauté du droit) ou non (respect des droits acquis, respect des attentes légitimes, doctrine du précédent) procèdent de l'idée générale de sécurité juridique ${ }^{154}$.

Bref, la doctrine canadienne ne considère toujours pas la sécurité juridique comme une norme juridique, mais comme un

d'UNIDROIT et le Code civil du Québec : variations et mutations " (2002) 36 R.J.T. 583 aux pp. 594, 595, 608.

150. En ce sens : Sylvio Normand, "La codification de 1866 : contexte et impact " dans Droit québécois et droit français: communauté, autonomie, concordance, Cowansville, Yvon Blais, 1993 à la p. 44; Esther Arroyo I Amayuelas, "Le Code civil catalan : choix, finalités et transplantations législatives du Code civil québécois " (2005) 46 C. de D. 271 à la p. 276. Pierre Larouche, supra note 127 à la p. 141.

151. En ce sens : Pierre Azard, "Le problème des sources du droit civil dans la province de Québec " (1966) 44 R. du B. can. 417 à la p. 456; Stéphane Bernatchez, "Les traces du débat sur la légitimité de la justice constitutionnelle dans la jurisprudence de la Cour suprême du Canada " (2005-2006) 36 R.D.U.S. 165 à la p. 230.

152. Côté, "souci de la sécurité ", supra note 127 à la p. 687.

153. Charpentier, supra note 127 à la p. 547.

154. Larouche, supra note 27 à la p. 141 . 
idéal qui permet d'expliquer voire de décrire ou de critiquer l'état du droit.

\section{B. Le discours du juge de la Cour suprême}

L'étude du discours des juges canadiens sera limitée en droit canadien aux juges de la Cour suprême du Canada. Soulignons que les tribunaux inférieurs évoquent également occasionnellement le concept de sécurité juridique dans leurs jugements ${ }^{155}$.

La première référence à l'expression "sécurité juridique " que nous avons recensée remonte à l'année 1989 lorsque la Cour suprême cita un ouvrage ${ }^{156}$ qui utilisait cette expression ${ }^{157}$. En 1992, la Cour suprême utilise pour la première fois l'expression dans le cadre de son argumentation ${ }^{158}$. Un an plus tard, dans

155. Par ex. Brochu c. Société des loteries du Québec (Loto-Québec), 2009 QCCS 5678 au par. 91 : "Évidemment, la jurisprudence en droit privé québécois demeure un guide important. La Cour supérieure a suivi généralement la voie tracée par la Cour d'appel et la Cour suprême du Canada pour des motifs qui sautent aux yeux des praticiens. Notamment, le principe de sécurité juridique, voulant que la règle de droit jurisprudentielle reflète pour les justiciables un certain degré de certitude, prévisibilité et stabilité raisonnable, exige des motifs sérieux pour qu'un tribunal s'écarte d'un courant de jurisprudence constant et bien établi (exemple: corriger une erreur, éviter une injustice, assurer le progrès et l'évolution du droit en le réformant et rechercher la véritable intention du législateur)"; Québec (P.G.) c. Corneau, 2010 QCCS 463 (CanLII) au par. 39 : "Dans ces conditions, bien que la règle du " stare decisis " n'existe pas en droit civil québécois, le principe de la sécurité juridique commande que le Tribunal suive le courant jurisprudentiel inspiré de l'arrêt Hétu, en l'absence de motif sérieux de s'en écarter".

156. Gilles Pépin et Yves Ouelette, Principes de contentieux administratif, 2e éd. Cowansville, Yvon Blais, 1982 à la p. 221

157. Chandler c. Alberta Association of Architects, [1989] 2 R.C.S. 848 au par. 42 [Chandler].

158. Tremblay c. Québec (Commission des affaires sociales), [1992] 1 R.C.S. 952 [Tremblay], le juge Gonthier écrit, au nom de la Cour, à la p. 968 : "[C]et objectif de cohérence répond à un besoin de sécurité des justiciables, mais également à un impératif de justice. Les décisions de la 
Perspectives pour un principe de sécurité juridique

(2009-10) 40 R.D.U.S. en droit canadien :

l'arrêt Domtar Inc. c. Québec (CALP), la Cour suprême du Canada a admis alors que la sécurité juridique est un "objectif du droit "159. Depuis, la sécurité juridique a été considérée par la Cour comme un objectif du droit pénal ${ }^{160}$ et du droit international privé ${ }^{161}$.

L'objectif de sécurité juridique a été rarement évoqué par la Cour suprême162 et il n'a jamais fait l'objet d'une définition claire ou d'une explication par le plus haut tribunal du Canada. La sécurité juridique constitue une sorte de critère qui est parfois évoqué explicitement sans qu'il fasse l'objet d'un exposé. L'évocation de la sécurité juridique dans la jurisprudence de la Cour suprême n'est pas significative en ce qu'elle se limite à la considérer comme un objectif ou un critère d'application de principe ou à titre d'élément d'une analyse beaucoup plus large. L'utilisation dans le discours des juges de la Cour suprême du concept de sécurité juridique demeure donc limitée et semble parfois fortuite. Dans tous les cas, la Cour n'a pas ressenti le besoin de définir le sens et les conséquences juridiques qui en découleraient.

L'introduction du concept de sécurité juridique dans le discours juridictionnel et doctrinal canadien n'a pas amené le droit canadien à reconnaître un statut formel à la sécurité juridique en droit positif. La doctrine canadienne n'a pas évolué d'une conception de la sécurité juridique en tant qu'objectif du droit vers une conception normative, comme l'a pourtant fait son homologue européen. Également, la Cour suprême n'attribue pas

Commission étant sans appel, c'est à la Commission elle-même qu'il revient de prévenir les incohérences décisionnelles ".

159. Domtar Inc. c. Québec (CALP), [1993] 2 R.C.S. 756 aux pp. 799-800.

160. Proulx c. Québec (P.G.), [2001] 3 R.C.S. 9 au par. 144.

161. GreCon Dimter Inc. c. J.R. Normand Inc., [2005] 2 R.C.S. 401 au par. 19 [GreCon].

162. Chandler, supra note 157; Tremblay, supra note 158; Domtar, supra note 159; Doucet-Boudreau c. Nouvelle-Écosse (Ministre de l'Éducation), [2003] 3 R.C.S. 3; Lefebure (Syndic de); Tremblay (Syndic de), [2004] 3 R.C.S. 326; Groupe HPS c. Bureau des services financiers, [2003] R.J.Q. 897; GreCon, supra note 161; Dell Computer Corp. c. Union des consommateurs, [2007] 2 R.C.S. 801. 
de statut normatif à la sécurité juridique contrairement au discours juridictionnel européen.

\section{Conclusion générale}

Notre étude du droit européen révèle que la reconnaissance par le juge européen et par la doctrine d'un statut de " principe " à la sécurité juridique a fait suite à une vaste littérature dans le discours doctrinal sur la "valeur" de sécurité juridique. L'exemple des juridictions européennes illustre le potentiel juridique du principe, c'est-à-dire son influence sur la pensée juridique et sur l'élaboration des règles de droit. En effet, "l'action de l'interprète conduit à modifier les représentations des concepts juridiques parce que sa parole est juricréatice " et parce que "le travail de la doctrine participe à la précompréhension et à l'élaboration des outils intellectuels d'interprétation des faits et des normes "163. D'ailleurs, plusieurs concepts nés dans la doctrine ont été ensuite reconnus par le pouvoir juridictionnel, comme revêtus d'un statut de règle ou de principe de droit positif.

La popularité croissante dans la doctrine de la notion de sécurité juridique et ses récentes concrétisations jurisprudentielles à titre de principe dans les droits nationaux et supranational européen permettent d'anticiper un renouvellement de l'intérêt relativement à la sécurité juridique en droit canadien. L'usage récent de la notion de sécurité juridique dans le discours

163. Boudot, supra note 15 à la p. 338; Gérard Cornu, "Réflexion finale " dans Théorie des actes du langage, éthique et droit, Paris, Presses Universitaires de France, 1986 à la p. 251; Philippe Jestaz, "La jurisprudence : réflexions sur un malentendu ", D. 1987. Chron. à la p. 17 : il y aurait en somme deux sources du droit : la loi pour l'essentiel; et à égalité de rang, quoiqu'avec un rayon d'action infiniment moindre, l'opinio juris ou la commune reconnaissance des juristes. Celle-ci donnerait force de droit et légitimité à des propositions linguistiques exprimées soit par un folklore anonyme (règle d'origine coutumière), soit à la rigueur par des sources prestigieuses ou représentatives (droit non légiféré d'origine diverse), soit surtout par une haute juridiction (jurisprudence proprement dite en qui s'absorbe plus ou moins les autres sources extralégales). 
Perspectives pour un principe de sécurité juridique

(2009-10) 40 R.D.U.S. en droit canadien :

des juristes canadiens exprime " une représentation nouvelle de la réalité " qui pourrait "par un effet de dépassement " "agir sur elle" et emporter l'émergence d'un principe de sécurité juridique ${ }^{164}$. Si "[l]e discours juridique est une parole créatrice, qui fait exister ce qu'elle énonce " 165 et qu'il " a un effet performatif dans la mesure où les arguments de la doctrine ont pour conséquences de convaincre que des concepts sont devenus des réalités "166, l'émergence du concept de sécurité juridique dans le discours juridique canadien pourrait se métamorphoser en une conception normative de la sécurité juridique.

Ainsi, en droit canadien, le recours à la notion de sécurité juridique et sa mise en forme discursive pourraient être le signe précurseur d'une éventuelle consécration en droit positif de la sécurité juridique. D’un point de vue hypothétique, la doctrine canadienne et le droit européen, qui contribuent à définir la sécurité juridique et s'appuient sur son autorité, pourraient influencer à terme la Cour suprême, qui, utilisant déjà l'expression, pourrait être tentée de consacrer dans sa jurisprudence le principe de sécurité juridique.

En particulier, il est possible que ce concept devienne à terme, un principe d'interprétation. Par exemple, en août 2009, dans le cadre d'une requête interlocutoire en Cour fédérale, un demandeur a récemment introduit le concept de sécurité juridique dans son discours à titre de principe devant guider l'interprétation des tribunaux, principe d'interprétation qui doit céder le pas au principe de démocratie dans certaines circonstances ${ }^{167}$. La Cour

164. Étienne Picard, "L'émergence des droits fondamentaux en France, dans Les droits fondamentaux - Une nouvelle catégorie juridique?" A.J.D.A. 1998, nº spécial juillet-août à la p. 6.

165. Pierre Bourdieu, Ce que parler veut dire, Paris, Fayard, 1982 à la p. 21 .

166. Boudot, supra note 15 à la p. 318.

167. Roger Collard c. La présidente d'élection du Conseil de Bande de Betsiamites, 2009 CF 812 au par. 40 : "Le demandeur note que le principe de délai de recours et de la prescription ont notamment pour fondements la stabilité et la sécurité juridique découlant de la rule of law. Or, en matière d'élection, l'objet de la loi est de préserver la confiance des citoyens envers les institutions démocratiques et d'assurer l'effectivité du 
suprême a déjà reconnu l'indépendance judiciaire, le fédéralisme, la primauté du droit, le constitutionnalisme, la démocratie et la protection des minorités à titre de principes implicites de l'ordre constitutionnel canadien ${ }^{168}$. La Cour a d'ailleurs expressément mentionné que cette liste n'est pas limitative. Ces principes constitutionnels implicites reconnus par la Cour suprême " ont un caractère prescriptif, au sens juridique du terme [...] bien que leur justiciabilité soit variable et puisse faire l'objet de débat "169. Ils "guident l'interprétation des dispositions explicites de la Constitution, de toute disposition législative et, généralement, de l'action gouvernementale "170. Ainsi, le droit canadien pourrait, à l'avenir, reconnaître le statut de principe implicite de l'ordre constitutionnel à la sécurité juridique.

Évidemment, le futur est incertain sur la forme que prendra le concept de sécurité juridique en droit canadien. L'on ne peut savoir avec certitude l'impact du discours des juristes sur l'évolution du droit sur cette question et la forme qu'aura, le cas échéant, la sécurité juridique171. Il est permis de croire que les juristes canadiens ne pourront éviter la délicate tâche de préciser l'état du droit relativement à la sécurité juridique.

principe de démocratie, deux principes qui doivent guider l'interprétation des tribunaux (voir Renvoi relatif à la sécession du Québec, [1998] 2 R.C.S. 217, 81 A.C.W.S. (3d) 798 aux par. 50, 61, 64 et 67). Le juste équilibre entre le principe de la démocratie et de la rule of law requiert la possibilité pour la présidente d'élection de proroger le délai pour contester l'élection en vertu du Code électoral pour des motifs exceptionnels ". Dans cette affaire, le Code électoral de la communauté autochtone de Betsiamites prévoyait que le délai pour interjeter appel de l'élection était de 14 jours. Le demandeur voulait faire reconnaître le pouvoir de déroger exceptionnellement à cette règle en cas d'impossibilité en fait d'agir.

168. Renvoi relatif à la sécession du Québec, [1998] 2 R.C.S. 217 aux par. 50, $61,64,67$.

169. Jean-François Gaudreault-Desbiens et Diane Labrèche, "Le contexte social du droit dans le Québec contemporain ", École du Barreau du Québec, Éthique, déontologie et pratique professionnelle, collection de droit, vol.1, Cowansville, Yvon Blais, 2007 à la p. 233.

170. Ibid.

171. Comparer Valembois, supra note 3 à la p. 461 : "[o]n ne peut prédire la survenue de sa cause occasionnelle ni les formes qu'elle prendra ". 